\title{
Label-free Quantitative Proteomics for Investigating the Therapeutic Mechanism of Sijunzi Decoction on Spleen Deficiency Syndrome
}

Qinghua Yan ( $\sim$ yanqinghua1980@163.com )

Xinxiang Medical University

\section{Shijiang Ma}

the Third Affiliated hospital of Xinxiang Medcial University

Hongli Chen

Xinxiang Medical University

Qiqing Zhang

Xinxiang Medical University

\section{Research}

Keywords: proteomics, sijunzi decoction, spleen deficiency syndrome, therapeutic mechanisms

Posted Date: February 24th, 2020

DOI: https://doi.org/10.21203/rs.2.24390/v1

License: (9) This work is licensed under a Creative Commons Attribution 4.0 International License. Read Full License 


\section{Abstract}

Background: Sijunzi decoction is a traditional Chinese medicine prescription which is used to treat spleen deficiency syndrome. But the therapeutic mechanism of Sijunzi decoction on spleen deficiency syndrome is unclear.

Methods: A rat model of reserpine-induced spleen deficiency syndrome was established. The therapeutic effects of Sijunzi decoction were assessed by histopathological examination and enzyme activity analysis. A label-free proteomics method was used to assess the changes in the endogenous protein. The differentially expressed proteins (DEPs) were analyzed using bioinformatics methods. Western blot was used to validate the proteomic data.

Results: Sijunzi decoction could significantly restore damaged liver tissue and enzymatic activities. DEPs of Sijunzi decoction-intervened spleen deficiency syndrome mainly regulated various metabolic processes involved in cellular responses to enzyme activity for catalysing, transforming and transferring. The four pathways (bile secretion, pyruvate metabolism, cysteine and methionine metabolism, and fatty acid metabolism) might be closely related with the intervention mechanism of Sijunzi decoction. Protein H2afx, interferon stimulated gene 15 protein, and signal transducers and activators of transcription1 proteins were predicted to play the important roles in Sijunzi decoction action networks.

Conclusions: Sijunzi decoction could exert intervention action by restoring DEPs involved in potential target pathways. Current results wish to provide novel perspectives for revealing the potential protein targets of Sijunzi decoction on spleen deficiency syndrome and facilitating its clinical usage.

\section{Background}

According to the theory of traditional Chinese medicine (TCM), the complete dysfunction of the spleen, and the lymphatic and pancreatic systems, as described in modern anatomy, is called spleen deficiency syndrome [1, 2]. Several studies have reported that spleen deficiency syndrome is related to digestive system diseases, energy metabolism, immune dysfunction of gastrointestinal motility, and stomach function [3]. Sijunzi decoction is a traditional Chinese herbal formula, first used in China during the Song dynasty. It is comprised of four types of herbs, Radix Ginseng, Rhizoma Atractylodes Macrocephala, Poria cocos and Glycyrrhiza uralensis [4]. Accumulated studies have demonstrated SJZD could enhance the body's immune function $[5,6]$. At the same time, it has numerous pharmacological effects such as promoting digestion and absorption, improving disturbances to the gut microbiota, regulating gastrointestinal motility, causing resistance to gastrointestinal mucosal injury, and enhancing intestinal mucosal immunity [7-9]. SJZD has been widely used to treat spleen deficiency syndrome. However, the therapeutic mechanisms are not yet well understood. Many scholars have studied the function of Sijunzi decoction based on a certain aspect of the body or a certain system. A comprehensive analysis evaluating the effect of Sijunzi decoction has yet to be conducted. 
The rapid growth of the proteomics field has resulted in an array of new tools for studying TCM and proteomic analysis has also facilitated the globalization of $\operatorname{TCM}[2,10,11]$. Being aware of the intrinsic relationship between TCM and systems biology, some researchers have already proposed that proteomics approaches may be helpful in validating TCM theories [12-14]. Proteomics research provides the basis for studying the action mechanisms of drugs, as well as providing a platform for comparing protein expression between different pathologies, and for screening novel targets for drug intervention $[15,16]$. In MS-based proteomics, isotope labelling and the label-free approach are two basic methods for quantitative analysis of protein expression $[17,18]$. In recent years, label-free proteomics methods have been considered as a promising alternative because of the reliability and validity. There are relatively few reports on the proteomics research of spleen deficiency syndrome. Li et al first reported the differentially expressed proteins and pathways of spleen-deficiency syndrome rats using isotope labelling proteomics methods [19]. Liu et a/ investigated the effect of traditional Chinese medicine Yi Tang Kang on metabolic syndrome of spleen deficiency with iTRAQ-based proteomics anslysis [20]. At present, there are almost no studies on spleen deficiency syndrome using label-free proteomics approaches.

It is unclear whether the pharmacological mechanisms of SJZD on spleen deficiency syndrome target multiple pathways. In this study, under the guidance of TCM theory of the spleen, and by reflecting on the concept of "wholeness", a characteristic subject of TCM, we integrated data from clinical symptoms monitoring, physiological and biochemical indices determinations, histopathological observations, and protein expression level quantification. To achieve the differentially expressed spectra of proteins using label-free proteomic technology was the primary focus of this study. Furthermore, the mechanism of action of Sijunzi decoction in spleen deficiency syndrome was further developed using bioinformatic analysis. This study takes a holistic approach to develop our understanding of the effect of Sijunzi decoction on spleen deficiency syndrome.

\section{Materials And Methods}

\section{The preparations of Sijunzi decoction}

Sijunzi decoction consisted of four crude herbs, Radix Ginseng (batch No. 401004713), Rhizoma Atractylodes Macrocephala (batch No. 002010401), Poria cocos (batch No. 002010301) and Glycyrrhiza uralensis (batch No. 003030601) at a ratio of 2:2:2:1 (W/W). All herbs were purchased from a local herbal shop (Tongrentang Herbal Co., Ltd., Xinxiang, China) and authenticated by the College of Traditional Chinese Medicine, Xinxiang Medical University, (Xinxiang, China). The preparation and manufacturing process of all herbs followed standard operating procedures according to a previous study [21]. The raw materials were socked in distilled water and boiled twice for $40 \mathrm{~min}$, respectively. The drug solution was filtered and concentrated in a water bath at $70^{\circ} \mathrm{C}$ to $80^{\circ} \mathrm{C}$. The final concentration was $2 \mathrm{~g}$ of crude drug materials $/ \mathrm{mL}$, and the solution was stored at $4{ }^{\circ} \mathrm{C}$. For the delivery of Sijunzi decoction using the drug gavage method, $1 \mathrm{~mL} / 100 \mathrm{~g}$ of body weight was delivered. The dosage of Sijunzi decoction used was the clinical dose converted into an experimental equivalent dose based on the standard body shape coefficient [22]. 
Thirty male Sprague Dawley 3-month-old rats with a body mass of $220 \pm 10 \mathrm{~g}$ were provided by the Laboratory Animal Center of Henan Province (Zhengzhou, China) (Rodent license No. SCXK 20100002). After the cages were separated, the animals were kept in sterilized rooms at a constant temperature (20 \pm $2{ }^{\circ} \mathrm{C}$ ) and humidity (50 $\left.\pm 20 \%\right)$, as well as natural circadian rhythm illumination. The rats were observed for 3 days to ensure they adapted to the environment and were healthy.

These rats were then randomly divided into three groups: the normal control group $(\mathrm{NC}, \mathrm{n}=10)$, the disease model group ( $M, n=10)$, and the Sijunzi decoction-treated group (SJZD, $n=10)$. The preparation of the animal model followed standard operating procedures according to a previous study [21].NC rats were given a subcutaneous injection of $0.2 \mathrm{~mL}$ normal saline daily at 9 am and an intragastric dose of 2 $\mathrm{mL}$ distilled water daily at $4 \mathrm{pm} . \mathrm{M}$ rats were given a daily subcutaneous injection of $0.6 \mathrm{mg} / \mathrm{kg}$ of reserpineat 9 am and an intragastric dose of $2 \mathrm{~mL}$ distilled water at $4 \mathrm{pm}$. Rats in the Sijunzi decoctiontreated group were given a subcutaneous injection of $0.6 \mathrm{mg} / \mathrm{kg}$ of reserpinedaily at 9 am and were given S Sijunzi decoction via gavage $(1 \mathrm{~mL} / 100 \mathrm{~g}$ of body weight) at $4 \mathrm{pm}$. The rats were treated daily for 14 days and weighed every morning. Successful modeling of spleen deficiency syndrome was confirmed by diarrhea, loss of appetite, weight loss, wilting, dull coat, and collapsing [23].

Animal welfare and experimental procedures were performed in accordance with the Guide for the Care and Use of Laboratory Animals (Ministry of Science and Technology of China, 2006) and were approved by the Animal Ethics Committee of Xinxiang Medical University.

\section{Sample collection}

After the model was finished, the rats were anesthetized using $10 \%$ chloral hydrate and dissected. Liver tissues were resected and divided into three parts. One part of the samples was fixed in $3.7 \%(\mathrm{v} / \mathrm{v})$ formalin solution for histopathological examination. The others were frozen in liquid nitrogen immediately and stored at $-80^{\circ} \mathrm{C}$ for further analysis (enzyme activity determination and proteomics analysis).

\section{Histopathological examination}

The same parts from resected liver tissues of each group were fixed in 3.7\% (v/v) formalin solution immediately and imbedded into paraffin wax. Sections were then stained with hematoxylin-eosin stain and examined under a biological microscope to observe any morphological differences.

\section{Enzyme activity analysis}

Rat livers were comminuted using liquid nitrogen after removal from the refrigerator. The activities of SDH and LDH in the liver tissues were analyzed using the SDH and LDH assay kits (Nanjing Jiancheng Biological Engineering Co., Ltd, Nanjing, China), respectively. 


\section{Protein extraction and Protein digestion with the FASP method}

The proteins from same parts of liver tissues were cut into pieces with clean scissors and lysed in an appropriate amount of SDT lysis buffer (SDS, dithiothreitol, Tris). Then, the liver lysate was ground repeatedly with a full-automatic sample frozen grinding machine (JXFSTPRP-III, Shanghai Jingxin Industrial Development CO., LTD, Shanghai, China) until completely pulverized. Samples were homogenized with a Vortex mixer (Digital Vortex-Genie 2; Scientific Industries, Inc., Bohemia, NY) and Ultrasonic treatments ( $100 \mathrm{~W}, 10$ s with 10 s rest, 10 times in total) were utilized. After $5 \mathrm{~min}$ of boiling in a water bath, the mixture was cooled to room temperature. After centrifugation, the supernatant of each sample was quantified using the fluorescence protein quantification method and analysed by SDSPAGE.

A modified protocol based on the previous method was used for protein digestion [24]. Approximately $300 \mu \mathrm{g}$ of protein extract from each sample was added into $200 \mu \mathrm{L}$ of urea (UA) buffer (8 M Urea, 150 $\mathrm{mM}$ Tris-HCl pH 8.5). After mixing, the mixture was transferred into Microcon $10 \mathrm{kD}$ ultra-fraction tube (Merck-Millipore, Germany) and then centrifuged at $14,000 \mathrm{~g}$ for $10 \mathrm{~min}$. The fluid in the bottom of the collecting pipe was discarded. $200 \mu \mathrm{L}$ of UA buffer were put into an ultra-fraction tube, rotated at 14000 rpm $\left(4{ }^{\circ} \mathrm{C}\right)$ for $15 \mathrm{~min}$. With the eluates discarded, $100 \mu \mathrm{L}$ of iodoacetamide (IAA, Bio-Rad) buffer (50 mM IAA in UA buffer) were pipetted to the ultra-fraction tubes, the ultra-fraction tubes were shaken at $600 \mathrm{rpm}$ for $1 \mathrm{~min}$, after which they were left at room temperature without light for $30 \mathrm{~min}$, and then centrifuged at $14,000 \times \mathrm{g}$ for $10 \mathrm{~min}$. To wipe off IAA, $100 \mu \mathrm{L}$ UA buffer was added to the ultra-fraction tubes, and the spun in the above conditions; this was repeated two times. After that, the ultra-fraction tubes were washed twice with $100 \mu \mathrm{L}$ of $50 \mathrm{mM} \mathrm{NH}_{4} \mathrm{HCO}_{3}$ and centrifuged at $14,000 \mathrm{~g}$ for $10 \mathrm{~min}$ at room temperature. Then, $40 \mu \mathrm{L}$ of trypsin buffer $\left(5 \mu \mathrm{L}\right.$ of trypsin diluted in $40 \mu \mathrm{L}$ of $50 \mathrm{mM} \mathrm{NH}_{4} \mathrm{HCO}_{3}$; trypsin from Promega, Madison, $\mathrm{Wl}$ ) was added into the ultra-fraction tubes. The samples were oscillated at 600 $\mathrm{rpm}$ for $1 \mathrm{~min}$. Then digestion was achieved at $37^{\circ} \mathrm{C}$ for 16 to $18 \mathrm{~h}$. The eluates were collected with new collecting tubes after centrifuging at $14,000 \mathrm{~g}$ for $10 \mathrm{~min}$. $25 \mu \mathrm{L} 25 \mathrm{mM} \mathrm{NH}_{4} \mathrm{HCO}_{3}$ was injected into each ultra-fraction tube. The ultra-fraction tubes were centrifuged at $14,000 \mathrm{~g}$ for $10 \mathrm{~min}$ after being oscillated at $600 \mathrm{rpm}$ for $1 \mathrm{~min}$. This was repeated one times. Then, the filtrate was incorporated and lyophilized. The freezing-drying peptides were dissolved with $50 \mu \mathrm{L}$ of a $0.1 \%$ trifluoroacetic acid (TFA, Sigma-Aldrich, USA). The fluorescence method (excitation wavelength of $295 \mathrm{~nm}$, emission wavelength of $350 \mathrm{~nm}$ ) was carried out to quantify the peptides and then analyzed by liquid chromatography-mass spectrometry/mass spectrometry (LC/MS/MS).

\section{LC-MS/MS analysis}

A $5 \mu \mathrm{g}$ sample of each group was analyzed in triplicate using LC/MS/MS. Analysis was conducted as previously described with few modifications [25]. The separation was done using an EASY-nLC1000 nanoflow HPLC (Thermo Scientific, USA). Flowing phases A and B were acetonitrile aqueous solution and acetonitrile solution, respectively, both containing $0.1 \%(\mathrm{v} / \mathrm{v})$ formic acid (Sigma-Aldrich). The chromatographic trap column (RP-C18, $20 \mathrm{~mm} \times 100 \mu \mathrm{m}, 5 \mu \mathrm{m}$; Thermo Scientific, USA) was equilibrated 
with $100 \%$ buffer $A$. The peptides were separated using the analysis column (RP-C18, $100 \mathrm{~mm} \times 75 \mu \mathrm{m}$, $3 \mu \mathrm{m}$; Thermo Scientific, USA) and trap column at a flow rate of $300 \mathrm{~nL} / \mathrm{min}$ with a gradient from 0 to $45 \%$ buffer B for 200 min, then $45 \%$ - 100\% buffer B for 200 - 216 min, and then 100\% buffer B for $216-240$ $\min$.

MS/MS was performed using a LTQ Orbitrap Velos Pro Mass Spectrometer (Thermo Finnigan, San Jose, CA). The mass spectrometer was operated in positive mode using a data-dependent acquisition method. The scanning range of the parent ions was $350-1800 \mathrm{~m} / \mathrm{z}$. After each full scan, the 15 most intense precursors were selected for fragmentation. The dynamic exclusion for MS/MS was set as $30 \mathrm{~s}$. The resolution of MS1 was 60,000 for $200 \mathrm{~m} / \mathrm{z}$. MS2 had unit mass resolution.

The mass spectrometry proteomics data have been deposited to the ProteomeXchange Consortium (http://proteomecentral. proteomexchange.org) via the iProX partner repository with the dataset identifier PXD015449 [26].

\section{Label-free analysis using Maxquant software}

Original LC-MS/MS files were imported into Maxquant (version 1.5.3) (https://www.maxquant.org/.) for database search and label free quantification (LFQ) analysis [27]. The database was downloaded from Uniprot (uniprot_rat_29389_20150420.fasta, containing 29,389 sequences, downloaded on April 20, 2018). Specific parameters were selected as follows: Main search ppm (6), Missed cleavage (2), MS/MS tolerance ppm (20), De-Isotopic (TRUE), Enzyme (Trypsin), Fixed modification (Carbamidomethyl (C)), Variable modification (Oxidation (M), Acetyl (Protein N-term)), Decoy database pattern (reverse), LFQ (TRUE), LFQ min ratio count (1), Match between runs (2 min), Peptide false discovery rate (0.01), and Protein false discovery rate $(0.01)$.

\section{Identification of DEPs}

DEPs were identified with a fold-change (FC) greater than 1.5 or less than 0.667 . These selected DEPs were used in a random variance model $t$-test performed using SPSS version 20.0 (IBM Corp., Armonk, NY, USA). Only proteins with a $p$ value $<0.05$ were considered to be significantly differentially expressed as previously reported $[28,29]$.

\section{Bioinformatics analysis}

A clustering analysis map was built using cluster 3.0 version 2.3 (Bio Fly Bioscience; www.bangfeibio.com/company) to identify DEPs with similar expression. Gene ontology (GO) annotation and Kyoto Encyclopedia of Genes and Genomes (KEGG) annotation for each protein were retrieved from the GO (http://www.geneontology.org/) and KEGG pathway database (http://www.genome.jp/Pathway) $[30,31]$, respectively. Proteins in the $\mathrm{GO}$ analysis were related at three ontologies-biological process (BP), cellular components (CC), and molecular functions (MF) [32]. Protein-protein interaction networks of the DEPs were analyzed by ingenuity pathway analysis (IPA) version 9.0 (Redwood City, CA, http://www. ingenuity.com ). 


\section{Western blot analysis}

Total proteins were extracted from samples of liver tissues $(100 \mathrm{mg})$, and $40 \mu \mathrm{g}$ proteins were separated by $12 \%$ SDS-PAGE and then transferred to PVDF membranes (Merck-Millipore, Germany). The membranes were blocked with $5 \%(\mathrm{w} / \mathrm{v})$ non-fat milk in TBST for $1 \mathrm{~h}$ and then incubated with the primary antibodies overnight at $4{ }^{\circ} \mathrm{C}$. After washing three times, the membranes were incubated with HRPconjugated secondary antibody for $1 \mathrm{~h}$ at room temperature. Subsequently, the membranes were washed and were visualized with the enhanced chemiluminescence reagent (Sigma Chemical Co, USA) and the images were generated using an AzureC600 instrument (Azure Biosystems, USA) and were calculated by Gray analysis. All the primary and secondary antibodies used in this western blot analysis came from Cell Signaling Technology (Cell Signaling Technology, USA).

\section{Statistical analysis}

In the proteomics experiments, statistically significant differences ( $p$ value $<0.05$ ) were analyzed between the $M$ and SJZD or M and NC group. In the enzyme activity assays, data were reported as the mean \pm standard error of mean (SEM) and statistically significant differences ( $p$ value $<0.05)$ were analyzed between the M and SJZD or M and NC group by one-way analysis of variance (ANOVA), followed by Tukey's multiple comparisons test. All statistical analysis was performed with SPSS 20.0 (SPSS, Armonk, New York, USA).

\section{Results}

\section{Body weight and viscera indices}

Body weight and viscera indices results are shown in Table 1. Compared with the NC group, the body weight and viscera (spleen and thymus) index of rats in the M group were significantly reduced ( $p$ value < $0.05)$ by the 14th day. Meanwhile, these indicators in the SJZD group were significantly increased ( $p$ value < 0.05 ) compared to the $M$ group. These results indicate that SJZD significantly reduces organ damage and increases the body weight and immunity of rats with spleen deficiency syndrome.

\section{Histopathological examination of liver tissues}

Liver histology was applied to assess the effect of SJZD on spleen deficiency syndrome (Fig. 1). No histopathological changes were found in the liver of the NC group. The liver tissue structure and cable structures were quite clear and the hepatocytes were normal. In the M group, the histopathology demonstrated there were extensive granular degeneration and vacuolar degeneration in liver tissues and a number of deposits of round lipid droplets around the central venous. In addition, the cytoplasm showed more severe cellular swelling. At the same time, there was extensive granular degeneration of the liver tissue, vacuole degeneration, central venous congestion phenomena, and the liver cell structures became fuzzy and disappeared in the liver tissue of the $\mathrm{M}$ group. Furthermore, neutrophils were found at the portal area of the liver. In the SJZD-treated group, a much smaller amount of fat droplets was 
observed compared to the $\mathrm{M}$ group, and there was only a small amount of existing granular degeneration in the liver cells and no congestion phenomena, which was similar to the NC group. This demonstrated that the animal model of spleen deficiency syndrome was successful.

\section{Enzyme activity analysis}

The SDH and LDH assay kits (Nanjing Jiancheng Biological Engineering Co., Ltd, China) were used for enzyme activity analysis. The SDH and LDH activity in the liver of each group can be seen in Fig. 2 . These results indicated that the activity of SDH was significantly decreased, while LDH activity was significantly increased ( $p$ value $<0.05$ ) in the M group compared to the NC group. However, the activity of SDH was significantly increased, and LDH activity was significantly decreased ( $p$ value $<.05)$ in the SJZD-treated group compared with the $\mathrm{M}$ group rats ( $p$ value $<0.05$ ). This demonstrated that the activity of SDH was significantly elevated and LDH activity reduced after SJZD treatment. Interestingly, the activities of SDH and LDH showed no significant difference between the SJZD group and the NC group ( $p$ value $>0.05$ ).

\section{Label-free proteomics analysis}

In this study, proteomics techniques were used to analyze the effect of SJZD on spleen deficiency syndrome and a total of 20,759 peptides and 3,021 proteins were identified. The DEPs were generated by comparing the M group with the NC group and the M group with the SJZD-treated group. With the screening criteria of DEPs (fold change $>1.5$ and $<0.67$; $p$ value $<0.05$ ), 272 DEPs were identified when the M group was compared to the NC group, with 128 upregulated and 144 downregulated proteins. When comparing the M group with the SJZD-treated group, 247 DEPs were identified, including 81 upregulated and 166 downregulated proteins. The DEPs identified in this study were listed in Additional file 1(Table S1-S4). As seen in Fig. 3, a Venn diagram analysis highlighted that there were 98 DEPs in common between the two comparisons.

\section{Cluster analysis of DEPs}

In order to further investigate the accuracy of the selected DEPs, clustering analysis was used to evaluate the DEPs. As seen in Fig.4, the DEPs identified in the M group and the SJZD-treated group can distinguish between the two groups. This indicates that the DEPs identified in this study may represent key differences between the two groups of samples.

\section{GO analysis}

The results of functional annotations of the DEPs using GO analysis are displayed in Fig. 5 . When the CC of these DEPs was analyzed, the majority of the DEPs were associated with the cytoplasm, cell membrane, and extracellular region (Fig.5A). In the MF analysis, the majority of the DEPs were associated with enzyme activity in catalyzing, transforming and transferring (Fig. 5B). The DEPs analyzed for their BP were found to be enriched in various metabolic processes (Fig.5C).

\section{KEGG pathway analysis}


To identify the potential target pathway of SJZD in spleen deficiency syndrome, we analysed the target pathway of the DEPs in rats with spleen deficiency syndrome and the target pathway of the DEPs in SJZD-treated rats with spleen deficiency syndrome. Pathway enrichment analysis of the DEPs was shown in Table 2 and Table 3. As seen in Table 2, the DEPs in rats with spleen deficiency syndrome were enriched in 11 biological pathways, namely, cysteine and methionine metabolism, bile secretion, retinol metabolism, drug metabolism-cytochrome P450, fatty acid metabolism, peroxisome, pyruvate metabolism, butanoate metabolism, phenylalanine metabolism, terpenoid backbone biosynthesis, and the AMPK signaling pathway. As seen in Table 3, the DEPs in SJZD-treated rats with spleen deficiency syndrome were significantly enriched in five biological pathways. These were bile secretion, pyruvate metabolism, cysteine and methionine metabolism, complement and coagulation cascades, and fatty acid metabolism. By comparing these two results, it was found that four metabolic pathways were common to both groups and these were bile secretion, pyruvate metabolism, cysteine and methionine metabolism, and fatty acid metabolism. It can be speculated that the four metabolic pathways are potential target pathways of SJZD treatment spleen deficiency syndrome.

\section{Protein interaction analysis}

The protein-protein interaction (PPI) network of the DEPs was shown in Fig. 6. In the PPI networks, nodes represent the identified DEPs and links represent correlation among different nodes. Proteins with high connectivity may be key points that affect metabolism or signal transduction pathways throughout the system. Data analysis indicated that some proteins in PPI networks could play key roles in SJZD intervention spleen deficiency syndrome and these included H2AFX, ISG15 and STAT1.

\section{Western blot verification of the differentially expressed proteins}

Three proteins H2AFX, ISG15 and STAT1 were selected to validate the results of label-free proteomics by performing western blot analyses. The results showed the expression levels of three proteins were significantly different between the $\mathrm{M}$ and NC groups or M and SJZD groups (Fig. 7A). We further analysed the bands based on the gray-level values of the three proteins. H2AFX was significantly upregulated in M group compared with the NC and SJZD groups, while ISG15 and STAT1 were significantly downregulated, consistent with the proteomics results (Fig. 7B).

\section{Discussion}

In this study, the results of body weight and viscera indices, enzyme activity assays, histopathological observations and proteomic analysis suggest that Sijunzi decoction has a protective effect on rats with spleen deficiency syndrome. Most important of all, the proteomic analysis revealed the potential therapeutic mechanism of Sijunzi decoction in rats with spleen deficiency syndrome.

In addition to being closely related to the digestion and absorption of nutrients, the function of the spleen is also closely related to material and energy metabolism. Studies have reported that the activity of enzymes related to energy metabolism in patients with spleen deficiency syndrome differ from that of 
healthy individuals [33]. SDH is one of the key enzymes that catalyzes oxidative phosphorylation reactions in the inner mitochondria of cells. The first dehydrogenase in the tricarboxylic acid cycle in the central metabolism of the body reflects the energy supply of oxidative phosphorylation in cells. Xue et al. found that SDH activity in spleen deficiency rats was significantly decreased, and was one of the important causes of digestive dysfunction [34]. LDH is the most active glycolytic enzyme in the liver, and also exists in the cytoplasm of all tissues in the body. LDH can catalyse lactic acid transformation into pyruvate, and therefore it can be used as a standard to predict the anaerobic metabolic capacity of the body. In this study it was found that compared with the NC group, the SDH activity in the liver of the M group was significantly decreased ( $p$ value $<0.05$ ), while the activity of LDH was significantly increased ( $p$ value $<0.05$ ). This indicates that aerobic oxidation metabolism was decreased, and anaerobic oxidation reaction was increased in the liver tissues of the rats with spleen deficiency syndrome. This is consistent with previous studies where increased LDH activity was observed in rats with spleen deficiency syndrome [9]. After treatment with Sijunzi decoction, the enzyme activities recovered back to normal levels. This suggests that Sijunzi decoction can significantly aid in restoring mitochondrial health caused by spleen deficiency syndrome in rats and thereby improve the energy supply level in the body.

In this study, pathological observations were carried out on liver tissues of model rats, and it was found that the liver tissues of rats with spleen deficiency syndrome had varying degrees of pathological changes, consistent with previous studies [35]. Additionally, these results are consistent with previous research on the pathological changes in spleen deficiency syndrome such as congestion, cell degeneration, atrophy and inflammatory cell infiltration in rat liver tissues [36].

Label free proteomics technology was employed to identify differentially expressed proteins, to study the mechanism of action of Sijunzi decoction in rats with spleen deficiency syndrome. In this study, 272 DEPs (128 proteins up-regulated, 144 proteins down-regulated) in rats with spleen deficiency syndrome were found when comparing the protein expression spectrum of the M group with the NC group. When comparing the $\mathrm{M}$ group to the SJZD group, 247 DEPs (81 proteins upregulated, 166 proteins downregulated) were found. Venn diagram showed there were 98 common DEPs. At the same time, we found that 81 proteins showed a reversal of the trend in the SJZD group, indicating Sijunzi decoction can adjust most of the DEPs and adjust their expression back to normal. It can be speculated that the common DEPs are potential target proteins of Sijunzi decoction when treating spleen deficiency syndrome. GO enrichment analysis showed that the DEPs in Sijunzi decoction intervention rats were mainly distributed in the cytoplasm, cell membrane and extracellular region. Additionally, the main function of the proteins was found to be in the activity of enzyme catalysis, transformation and transfer as well as other various metabolic processes. By comparing the target pathways in rats with spleen deficiency syndrome and Sijunzi decoction intervention rats with spleen deficiency syndrome, it was revealed that the mechanism of action of Sijunzi decoction was mainly through the regulation of four pathways (bile secretion, pyruvate metabolism, cysteine and methionine metabolism, and fatty acid metabolism). 
The bile secretion pathway is an important secretion pathway in the body. The secretion and excretion of bile are regulated by a variety of bodily fluids, with gastrin promoting the secretion of bile [37]. Our pharmacodynamic studies showed that gastrin content in the blood of rats with spleen deficiency syndrome decreased significantly compared with that of the NC group ( $p$ value $<0.05)$. This suggests that the decrease of gastrin inhibits bile secretion, hindering the digestion and absorption of fats and vitamins. Fat metabolism in rats with spleen deficiency syndrome was restrained, leading to a shortage of nutrients and energy for the body, and consequently, rats with spleen deficient syndrome appeared thin and tired. This is consistent with the results of pharmacodynamics studies [38]. In the bile secretion pathway, the expression of solute carrier organic anion transporter family member 1A4 and bile salt export pump were up-regulated. The expression of ATP-binding cassette and neutral cholesterol ester hydrolase 1 were down-regulated. This indicates that bile secretion is dysfunctional in the liver of rats with spleen deficiency syndrome, leading to the inhibition of digestion and absorption functions. After Sijunzi decoction intervention intervention, gastrin content was found to be increased and bile secretion returned to normal. Additionally, DEPs (ATP-binding cassette, neutral cholesterol ester hydrolase 1) returned to normal, so that bile secretion in the liver was closer to normal and digestion and absorption ability were stable.

The cysteine and methionine metabolism pathway is a very important amino acid metabolism pathway in the body. Methionine and its metabolites have antioxidant capacities and maintain the redox state in cells. Cysteine and methionine metabolism can produce pyruvate in the body through a series of reactions. Pyruvate, the metabolic hub, enters many different metabolic pathways. There were four upregulated DEPs in cysteine and methionine metabolic pathways of the liver of rats with spleen deficiency syndrome $(p$ value $<0.01$ ), including L-serine dehydratase /L-threonine deaminase, aspartate aminotransferase, tyrosine aminotransferase, and S-methyl methionine-homocysteine Smethyltransferase BHMT2. This suggested that the metabolism of cysteine and methionine in liver tissues of rats with spleen deficiency syndrome was enhanced, indicating that the body of rats with spleen deficiency syndrome was under oxidative stress. Oxidative stress damages cells and causes their dysfunction, which leads to viscera damage and all kinds of metabolic disturbances. The expression of these proteins was significantly down-regulated after intervention with Sijunzi decoction ( $p$ value $<0.05)$, therefore highlighting that the metabolism of cysteine and methionine was gradually restored to normal in the liver.

Pyruvate acid is an important intermediate product in metabolic processes. Accordingly, pyruvate metabolic disorders disrupt a variety of metabolic processes in the body. In this study, the expression of L-lactate dehydrogenase $B$ chain and acetyl-CoA carboxylase 1 were upregulated in the $\mathrm{M}$ group, but the glyoxylate reductase/hydroxypyruvate reductase (GRHPR) protein was downregulated, indicating that the rats with spleen deficiency syndrome were suffering from pyruvate metabolic disorders. It can be speculated that there were three major nutrient disorders in spleen deficiency rats due to the changes in pyruvate metabolism, leading to further deficiency of nutrients and body emaciation. This is also consistent with the results of the physical indicators in this study. In addition, pyruvate is the energy source of mitochondria, and changes to pyruvate metabolism leads to functional changes in the 
mitochondria and a reduction in the energy supply to the body [39]. This was also consistent with the results of the enzymatic indicators of energy metabolism in this study. After treatment with Sijunzi decoction, pyruvate metabolism was restored to normal, and this then meant that all metabolic processes related to pyruvate metabolism were also restored to normal.

One of the functions of fatty acids in animals is to produce energy in the form of ATP. Compared with other nutrient carbohydrates and proteins, complete oxidation of fatty acids produces the most ATP energy [40]. In the mitochondria, fatty acids are degraded into acetyl-CoA by $\beta$-oxidation. On the other hand, acetyl-CoA can also synthesize fatty acids through the synthesis pathway of fatty acids in the cytoplasm. The two pathways are mutually inhibitory. Studies have showed that acetyl-CoA carboxylase can prevent acetyl-CoA produced from fatty acid $\beta$-oxidation entering the fatty acid synthesis pathway [40]. The expression of acetyl-CoA carboxylase was found to be up-regulated in the tissues of liver from the $\mathrm{M}$ group in this study, and the synthesis of fatty acids was inhibited. Therefore, due to the body's demand for energy, the breakdown of fatty acids was increased and fat was consumed in much larger quantities, resulting in the emaciation of the body. After treatment with Sijunzi decoction intervention, the expression of acetyl-CoA carboxylase decreased and fatty acid metabolism was returned to normal.

By constructing protein interaction networks represented by nodes and links, effective information of proteins can be extracted from different system scales and comprehensive information that cannot be obtained by a single protein is obtained. Potential protein targets of Sijunzi decoction intervention treatment spleen deficiency syndrome were predicted by PPI networks. ISG15, one of our potential protein targets, is known to be involved in the immune response, and has been proved to exert antiviral activity against a variety of viruses [41]. STAT1 is necessary for congenital immunity of the body. In addition, it can also act as a cell growth inhibitor and apoptosis activator. In this study, the expression of ISG15 and STAT1 were found to be downregulated in the liver tissue of $M$ group rats, resulting in immune system dysregulation. Protein H2AFX levels are associated with damage to DNA in cells [42]. The expression levels of H2AFX were found to be up-regulated in M group rats, which suggested the relevant DNA in the cell may be damaged in rats with spleen deficiency syndrome. After treatment with Sijunzi decoction, the expression of ISG15, STAT1 and H2AFX were called back. ISG15, STAT1 and H2AFX were detected by western blots and further verified the results of proteomics.

\section{Conclusions}

In conclusion, Current study demonstrated that Sijunzi decoction could remarkably improve spleen deficiency syndrome induced by reserpine. Liver proteomics speculated that bile secretion, pyruvate metabolism, cysteine and methionine metabolism, and fatty acid metabolism are potential target pathways of Sijunzi decoction intervention spleen deficiency syndrome. Bioinformatics analysis demonstrated that Sijunzi decoction could exert intervention action by restoring DEPs involved in potential target pathways. Current results wish to provide novel perspectives for revealing the potential protein targets of Sijunzi decoction on spleen deficiency syndrome and facilitating its clinical usage. 


\section{Abbreviations}

SDH: Succinate dehydrogenase; LDH: Lactic dehydrogenase; DEPs: Differentially expressed proteins; GO: Gene ontology; KEGG: Kyoto Encyclopedia of Genes and Genomes; H2AFX: Protein H2afx; ISG15: Interferon stimulated gene 15 protein; STAT1: Signal transducers and activators of transcription 1.

\section{Declarations}

\section{Acknowledgements}

We thank International Science Editing for providing language editing services for this manuscript.

\section{Publication statement}

The contents of this manuscript have not been published or submitted for publication elsewhere.

\section{Consent for publication}

Not applicable.

\section{Authors' contributions}

Qinghua Yan designed study, performed experiments, conducted data analysis and prepared manuscript. Shijiang Ma, contributed to experimental design and assisted with data interpretation. Qiqing Zhang helped with study design, data interpretation and manuscript preparation. Hongli Chen contributed to data interpretation and manuscript preparation. All authors read and approved the finally manuscript.

\section{Funding}

This work was supported by Key Scientific Research Projects of Higher Education Institutions of Henan Province (20A180002) and the Doctoral Scientific Research Foundation of Xinxiang Medical University (505304).

\section{Availability of data}

The mass spectrometry proteomics data have been deposited to the ProteomeXchange Consortium (http://proteomecentral. proteomexchange.org) via the iProX partner repository with the dataset identifier PXD015449. The datasets used and/or analyzed during the current study are available from the corresponding author on reasonable request.

\section{Ethics approval and consent to participate}

Animal welfare and experimental procedures were performed in accordance with the Guide for the Care and Use of Laboratory Animals (Ministry of Science and Technology of China, 2006) and were approved 
by the Animal Ethics Committee of Xinxiang Medical University. The manuscript does not contain human studies.

\section{Competing interests}

The authors declare that they have no competing interests

\section{References}

1. Xiong B, Qian HN. Effects of sijunzi decoction and yupingfeng powder on expression of janus kinasesignal transducer and activator of transcription signal pathway in the brain of spleen-deficiency model rats. J Tradit Chin Med. 2013;2013:78-4.

2. Zhang L, Wu Y, Zu XP, Xie HS, Li HK, Li YP, Zhang WD. Integrative metabolic and microbial profiling on patients with Spleen-yang-deficiency syndrome. Sci Rep. 2018;8. DOI: 10.1038/s41598-018-24130-7.

3. Piao S, Tan S, Li K, Zhan H, Xiao X, Guo J. UPLC-QTOF/MS-Based Lipidomic Profiling of Liver QiStagnation and Spleen-Deficiency Syndrome in Patients with Hyperlipidemia. Evid-Based Compl Alt. 2018. Article ID: 4530849.

4. Wang YY, He S, Cheng XC, Lu YX, Zou YP, Zhang QL. UPLC-Q-TOF-MS/MS fingerprinting of Traditional Chinese Formula SiJunZiTang. J Pharmaceut Biomed. 2013;80: 24-33.

5. Guan ZB, Wu J, Wang CC, ZhangF, Wang YA, Wang M, Zhao M, Zhao CJ. Investigation of the preventive effect of Sijunzi decoction on mitomycin C-induced immunotoxicity in rats by (1)H NMR and MS-based untargeted metabolomic analysis. J Ethnopharmacol. 2018;210: 179-91.

6. Chen JM, Yang TT, Cheng TS, Hsiao TF, Chang PMH, Leu JY, Wang FS, Hsu SL, HuangCY, Lai JM. Modified Sijunzi decoction can alleviate cisplatin-induced toxicity and prolong the survival time of cachectic mice by recovering muscle atrophy. J Ethnopharmacol. 2019;233:47-55.

7. Kang A, Guo JR, Zhang Z, Wang XL. Simultaneous quantification of ten active components in traditional Chinese formula Sijunzidecoction using a UPLC-PDA method. J Anal Methods Chem. 2014;2014. Article ID: 570359.

8. Yu BB, Zhou CX, Zhang JY, Ling Y, Hu QF, WangY, Bai KK. Latest study on the relationship between pathological process of inflammatory injury and the syndrome of spleen deficiency and fluid retention in Alzheimer's disease. Evid-Based Compl Alt. 2014;2014. Article ID: 743541.

9. Yu XY, Cui ZG, Zhou ZL, Shan T, Li DH, Cui NQ. Si-jun-zi decoction treatment promotes the restoration of intestinal function after obstruction by regulating intestinal homeostasis. Evid-Based Compl Alt. 2014;2014, DOI: 10.1155/2014/928579.

10. Zhang AH, Zhou XH, Zhao HW, Zou SY, Ma CW, Liu Q, Sun H, Liuc L, Wang XJ. Metabolomics and proteomics technologies to explore the herbal preparation affecting metabolic disorders using high resolution mass spectrometry. Mol Biosyst. 2017;13:320-9.

11. Zhang K, He MY, Su D, Pan X, Zhang H, Yang JY, Wu CF. Quantitative proteomics reveal antidepressant potential protein targets of xiaochaihutang in corticosterone induced model of 
depression. J Ethnopharmacol. 2018;231:438-45.

12. Sun SJ, Dai JY, Wang WY, Cao HJ, Fang JW, Hu YY, Su SB, Zhang YY. Metabonomic evaluation of ZHENG differentiation and treatment by fuzhenghuayu tablet in hepatitis-B-caused cirrhosis. EvidBased Compl Alt. 2012. Article ID: 453503.

13. Ji Q, Zhu FS, Liu X, Li Q, Su SB. Recent Advance in Applications of Proteomics Technologies on Traditional Chinese Medicine Research. Evid-Based Compl Alt. 2015. Article ID 983139.

14. Wei JY, Guo FF, Zhang MY, Xian M, Wang T, Gao J, Wu H, Song L, Zhang Y, Li D, Yang H, Huang L, Signature-oriented investigation of the efficacy of multicomponent drugs against heart failure. FASEB J. 2019;33:1-12.

15. Tao ZG, Meng X, Han YQ, Xue MM, Wu SF, Wu P, Yuan YZhu Q, Zhang TJ, Wong CCL. Therapeutic Mechanistic Studies of ShuFengJieDu Capsule in an Acute Lung Injury Animal Model Using Quantitative Proteomics Technology. J Proteome Res. 2017;16:4009-19.

16. Wang H, Wu G, Park HJ, Jiang PP, Sit WH, Van Griensven LJ, Wan JMF. Protective effect of phellinus linteus polysaccharide extracts against thioacetamide-induced liver fibrosis in rats: a proteomics analysis. Chin Med-UK. 2012;7:23.

17. Ong SE, Blagoev B, Kratchmarova I, Kristensen DB, Steen H, Pandey A, Mann M. Stable isotope labeling by amino acids in cell culture, SILAC, as a simple and accurate approach to expression proteomics. Mol Cell Proteom. 2002;1:376-86.

18. Gautier V, Mouton-Barbosa E, Bouyssie' D, Delcourt N, Beau M, Girard JP, Cayrol C, Burlet-Schiltz O, Monsarrat B, Gonzalez de Peredo A. Label-free quantification and shotgun analysis of complex proteomes by one-dimensional SDS-PAGE/NanoLCMS evaluation for the large scale analysis of inflammatory human endothelial cells. Mol Cell Proteomics Mcp. 2012;11:527-39.

19. Li C, Zhao RH, Xie M, Guo ZG, Sun W. Proteomics analysis of liver proteins from rats with spleendeficiency syndrome induced by chronic improper diet consumption and fatigue. J Tradit Chin Med Sci. 2017;4:113-126.

20. Liu XX, Shi Y. Intervention effect of traditional Chinese medicine Yi Tang Kang on metabolic syndrome of spleen deficiency. Asian Pac J Trop Med. 2015;8:162-8.

21. Yan QH, Mao HL, Wei YM. Elucidation of mechanism of Si-jun-zi decoction-induced reversal of spleen deficiency syndrome in rats by LC-Q-TOF/MS metabolomics. Trop J Pharm Res. 2017;16:525-33.

22. Yu WG, Lu B, Zhang HW, Zhang YX. J. Yan, Effects of the Sijunzi decoction on the immunological function in rats with dextran sulfate-induced ulcerative colitis. Biomed Rep. 2016;5:83-6.

23. Wang RJ, Peng Y, Meng H, Li XB. Protective effect of polysaccharides fractions from Sijunzi decoction in reserpine-induced spleen deficiency rats, RSC Adv. 2016;6:60657-65.

24. Wiśniewski JR. Quantitative evaluation of filter aided sample preparation (FASP) and multienzyme digestion FASP protocols. Anal Chem. 2016;88:5438-43.

25. Luber CA, Cox J, Lauterbach H, Fancke B, Selbach M, Tschopp J, Akira S, Wiegand, Hochrein MH, O'Keeffe, Mann M. Quantitative proteomics reveals subset-specific viral recognition in dendritic cells. Immunity. 2010;32:279-89. 
26. Ma J, Chen T, Wu S, Yang C, Bai M, Shu K, Li K, Zhang G, Jin Z, He F, Hermjakob H, Zhu Y. iProX: an integrated proteome resource. Nucleic Acids Res. 2019;47:D1211-7.

27. Cox J, Mann M. MaxQuant enables high peptide identification rates, individualized p.p.b.-range mass accuracies and proteomewide protein quantification. Nat biotechnol. 2008; 26:1367-72.

28. Zhan C, Yan L, Wang L, Jiang W, Zhang Y, Xi J, Jin Y, Chen L, Shi Y, Lin Z, Wang Q. Landscape of expression profiles in esophageal carcinoma by the cancer genome atlas data. Dis Esophagus. 2016;29:920-8.

29. Yan L, Zhan C, Wu JH, Wang SZ. Expression profile analysis of head and neck squamous cell carcinomas using data from the cancer genome atlas. Mol Med Rep. 2016;13:4259-65.

30. Kanehisa M, Sato Y, Kawashima M, Furumichi M, Tanabe M. KEGG as a reference resource for gene and protein annotation. Nucleic Acids Res. 2016;44:D457-62.

31. Kanehisa M, Goto S. KEGG: Kyoto Encyclopedia of Genes and Genomes. Nucleic Acids Res. 2000;28:27-30.

32. Harris MA, Clark J, Ireland A, Lomax J, Ashburner M, Foulger R, Eilbeck K, Lewis S, Marshall B, Mungall C, Richter J, Rubin GM, Blake JA, Bult C, Dolan M, Drabkin H, Eppig JT, Hill DP, Ni L, Ringwald M, Balakrishnan R, Cherry JM, Christie KR, Costanzo MC, Dwight SS, Engel S, Fisk DG, Hirschman JE, Hong EL, Nash RS, Sethuraman A, Theesfeld CL, Botstein D, Dolinski K, Feierbach B, Berardini T, Mundod i S, Rhee SY, Apweiler R, Barrell D, Camon E, Dimmer E, Lee V, Chisholm R, Gaudet P, Kibbe W, Kishore R, Schwarz EM, Sternberg P, Gwinn M, Hannick L, Wortman J, Wood V, de la Cruz N, Tonellato P, Jaiswal P, Seigfried T, White R. The gene ontology (GO) database and informatics Resource. Nucleic Acids Res. 2004;32:D258-61.

33. Zhang NW, Guo SN, Li HR, Li JF, Xu XL, Wan CR, Zhao H, Liu FQ, Zan JL, Wang B, Xu JQ. Effects of sijunzi decoction on small intestinal $T$ lymphocyte subsets differentiation in reserpine induced spleen deficiency rats. Anim J Vet Adv. 2013;12:1574-82.

34. Laurenti G, Tennant DA. Isocitrate dehydrogenase (IDH), succinate dehydrogenase (SDH), fumarate hydratase $(\mathrm{FH})$ : three players for one phenotype in cancer. Biochem Soc T. 2016;44:1111-6.

35. Ding YJ, Wang BC, Wen CC, Sun CY, Lee HH, Lee FP, Yang LL, Chen YH. Evaluation of the teratogenic effects of three traditional Chinese medicines, Si Jun Zi Tang, Liu Jun Zi Tang and ShenlingBaizhu San, during zebrafish pronephros development. J Toxicol Pathol. 2015;28:141-9.

36. Zan JL, Zhou B, Jia D, Xu XL, Wan CR, Wang N, Liu FH. Pathological changes of hepatic tissue in spleen deficiency rats. J Beijing Univ Agr. 2012;27:43-6.

37. Zhu M, College Physiology. 4th ed, BeiJing: High Education Press. 2017.

38. Yan QH, Mao HL, Guan JY. Metabolomics analysis reveals the mechanisms of the effect of Sijunzi decoction on spleen deficiency syndrome in a rat model. Microchem J. 2020;152:104422-32.

39. Meirleir LD, Disorders of pyruvate metabolism. Handbook Clin Neurol. 2013;113:1667-73.

40. Calder PC. Fatty acid metabolism. Encyclopedia Food Health. 2016;39:632-44. 
41. Haas AL, Ahrens $P$, Bright PM, Ankel H. Interferon induces a 15-kilodalton protein exhibiting marked homology to ubiquitin. J Biol Chem. 1987;262:11315-23.

42. Chowdhury D, Keogh MC, Ishii H, Peterson CL, Buratowski S, Lieberman J. $Y-H 2 A X$ Dephosphorylation by Protein Phosphatase 2A Facilitates DNA Double-Strand Break Repair. Mol Cell. 2005;20:801-9.

\section{Tables}

Table 1. Body weight and viscera indices results (mean \pm SD) $(n=10)$.

\begin{tabular}{llll}
\hline Group & $\begin{array}{l}\text { Body weight } \\
\text { [on the 14 th day口 }\end{array}$ & Spleen index & Thymus index \\
\hline NC & $310 \pm 15.570$ & $0.275 \pm 0.153$ & $0.091 \pm 0.041$ \\
M & $142 \pm 10.210^{*}$ & $0.201 \pm 0.074^{*}$ & $0.062 \pm 0.021^{*}$ \\
SJZD & $298 \pm 18.290^{\#}$ & $0.271 \pm 0.167^{\#}$ & $0.084 \pm 0.018^{\#}$ \\
\hline
\end{tabular}

* represent significantly different compared to the normal control group ( $p$ value $<0.05$ ), \# represent significantly different compared to the model group ( $p$ value $<0.05$ ).

Table 2. KEGG enrichment analysis of differentially expressed proteins (DEPs) of spleen deficiency syndrome. 


\begin{tabular}{|c|c|c|c|c|c|c|}
\hline ID & ID.Name & $\begin{array}{l}\text { Genes.in. } \\
\text { Category }\end{array}$ & Test\% & Ref\% & $P$. value & $\begin{array}{l}- \text { Log } \\
P \text { value }\end{array}$ \\
\hline 00270 & $\begin{array}{l}\text { Cysteine and methionine } \\
\text { metabolism }\end{array}$ & 7 & 0.055556 & 0.005573 & $2.46 \mathrm{E}-05$ & 4.609064893 \\
\hline 04976 & Bile secretion & 7 & 0.064815 & 0.011146 & 0.000181 & 3.742321425 \\
\hline 00830 & Retinol metabolism & 6 & 0.051423 & 0.02438 & 0.000064 & 4.193820026 \\
\hline 00982 & $\begin{array}{l}\text { Drug metabolism } \\
\text { cytochrome P450 }\end{array}$ & 6 & 0.052489 & 0.022412 & 0.000684 & 3.164943898 \\
\hline 01212 & Fatty acid metabolism & 5 & 0.048691 & 0.016321 & 0.000078 & 4.107905397 \\
\hline 04146 & Peroxisome & 5 & 0.046753 & 0.014832 & 0.000036 & 4.443697499 \\
\hline 00620 & Pyruvate metabolism & 4 & 0.037623 & 0.028641 & 0.000021 & 4.677780705 \\
\hline 00650 & Butanoate metabolism & 4 & 0.036542 & 0.018763 & 0.000065 & 4.187086643 \\
\hline 00360 & Phenylalanine metabolism & 3 & 0.029864 & 0.017543 & 0.000032 & 4.494850022 \\
\hline 00900 & $\begin{array}{l}\text { Terpenoid } \\
\text { biosynthesis }\end{array}$ & 3 & 0.017643 & 0.008564 & 0.000079 & 4.102372909 \\
\hline 04152 & AMPK signaling pathway & 3 & 0.013563 & 0.0086421 & 0.000098 & 4.008773924 \\
\hline
\end{tabular}

Table 3. KEGG enrichment analysis of differentially expressed proteins (DEPs) of Sijunzi decoction intervention spleen deficiency syndrome.

\begin{tabular}{|c|c|c|c|c|c|c|}
\hline ID & ID.Name & $\begin{array}{l}\text { Genes.in. } \\
\text { Category }\end{array}$ & Test\% & Ref\% & $P$. value & $\begin{array}{l}-\mathrm{Log} \\
\text { ( } P \text { value) }\end{array}$ \\
\hline 00982 & Bile secretion & 6 & 0.0567051 & 0.0111346 & 5.087342 & 6.114396 \\
\hline 00620 & Pyruvate metabolism & 2 & 0.0352689 & 0.0021684 & $2.86 \mathrm{E}-04$ & 3.543027 \\
\hline 00270 & $\begin{array}{l}\text { Cysteine and methionine } \\
\text { metabolism }\end{array}$ & 5 & 0.0505051 & 0.0055728 & 0.0001975 & 2.25393 \\
\hline 04610 & $\begin{array}{l}\text { Complement and coagulation } \\
\text { cascades }\end{array}$ & 7 & 0.0707071 & 0.0106811 & 7.96E-05 & 1.9713834 \\
\hline 01212 & Fatty acid metabolism & 4 & 0.0808081 & 0.0126935 & $3.21 \mathrm{E}-05$ & 1.8964187 \\
\hline
\end{tabular}




\section{Figures}
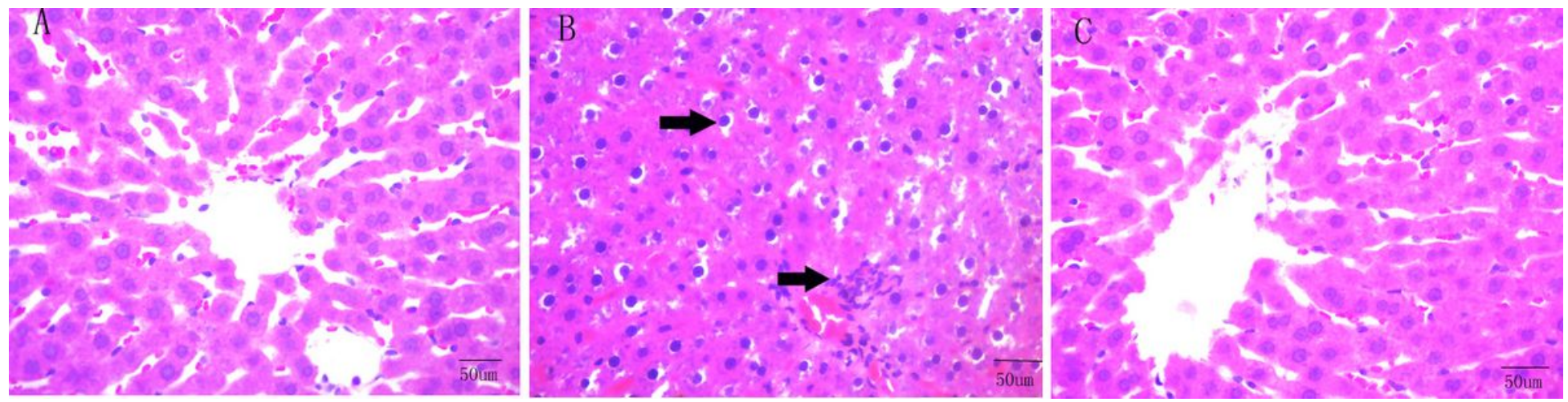

Figure 2

Representative HE-stained sections of liver in every group (400 ×). (A) the normal control group. (B) the model group. (C) SJZD-treated group. The arrows indicate pathological cells.
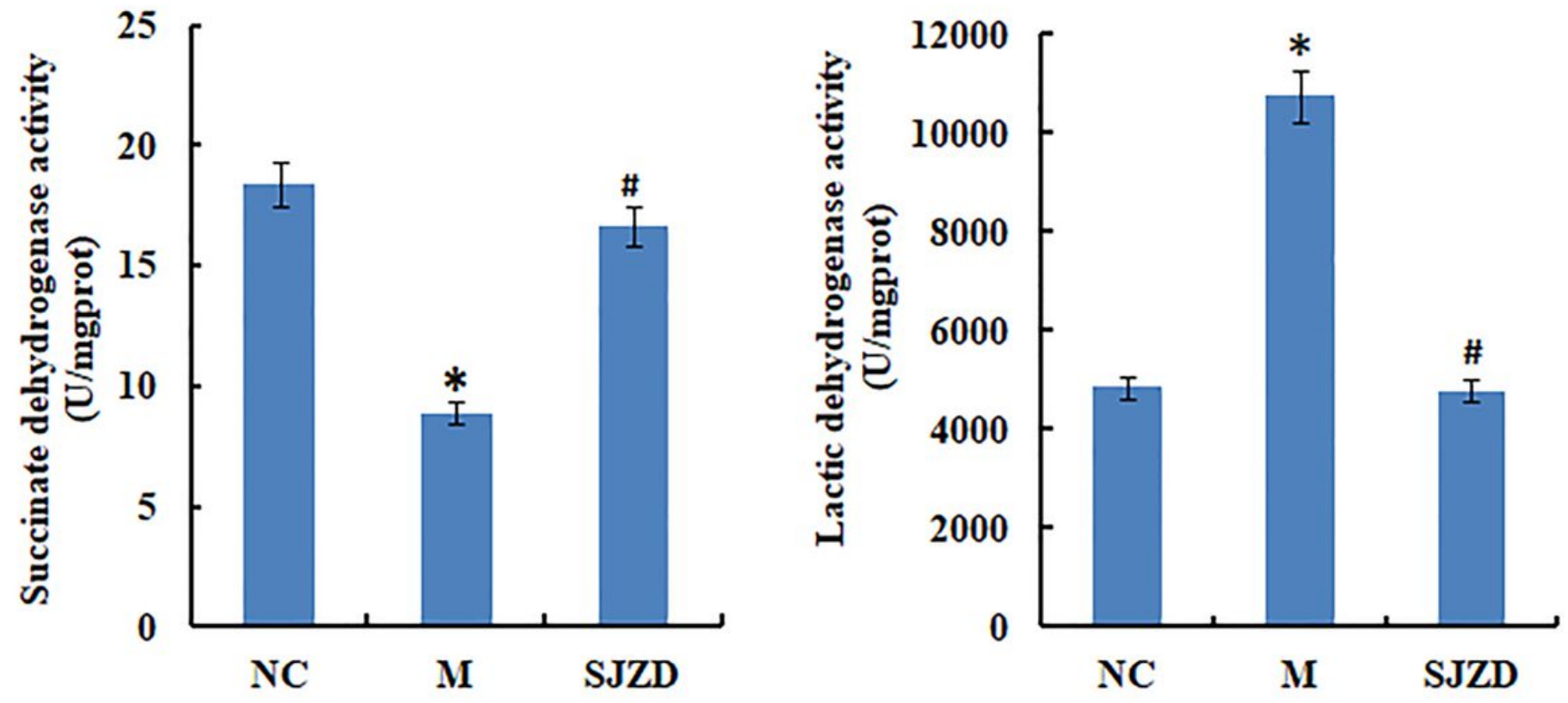

Figure 4

Succinate dehydrogenase (SDH) and lactate dehydrogenase (LDH) activity levesl in the liver of rats in each group (mean $\pm S D$ ) * represent significantly different compared to the normal control group ( $p$ value $<0.05$ ); ** represent highly significantly different compared to the normal control group ( $p$ value $<0.01)$; \# represent significantly different compared to the model group ( $p$ value $<0.05)$. 


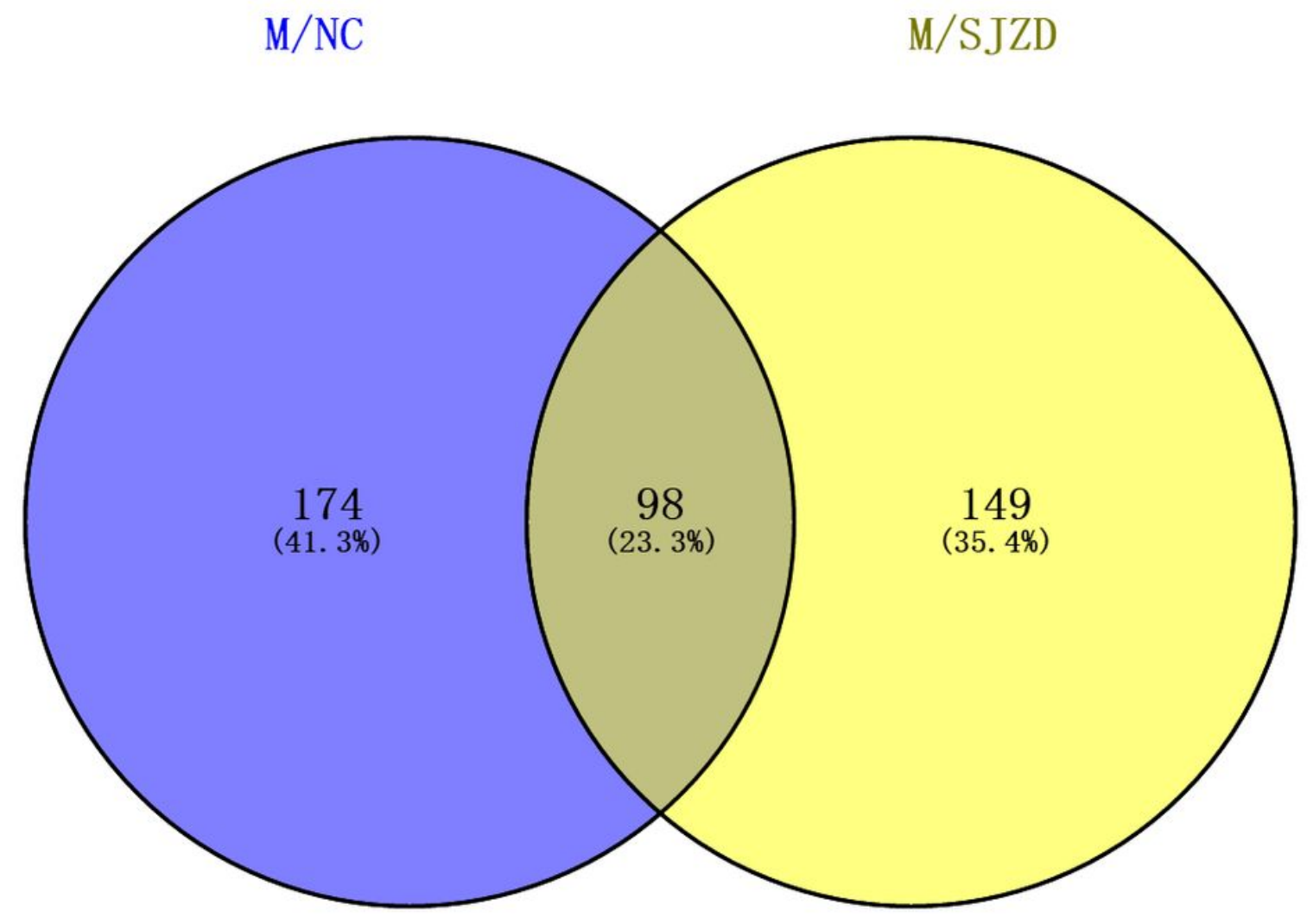

Figure 6

Wayne figure analysis of differentially expressed proteins (DEPs). The blue circle represents 272 DEPs identified by comparing the model with normal control groups; the yellow circle represents 247 DEPs identified by comparing the model with Sijunzi decoction-treated groups. 


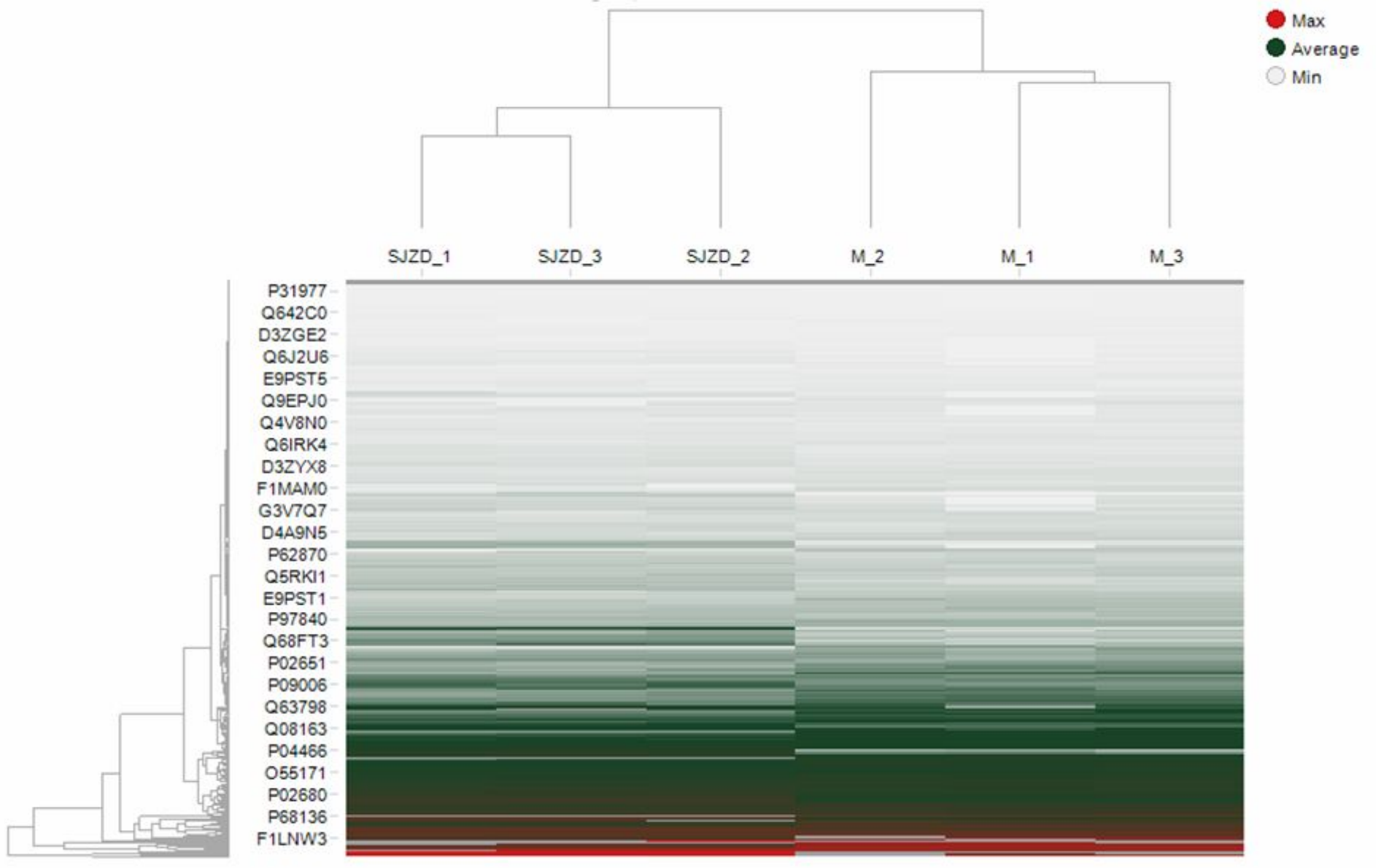

Figure 8

Cluster analyses of differentially expressed proteins (DEPs) (model vs. Sijunzi decoction-treated group) 

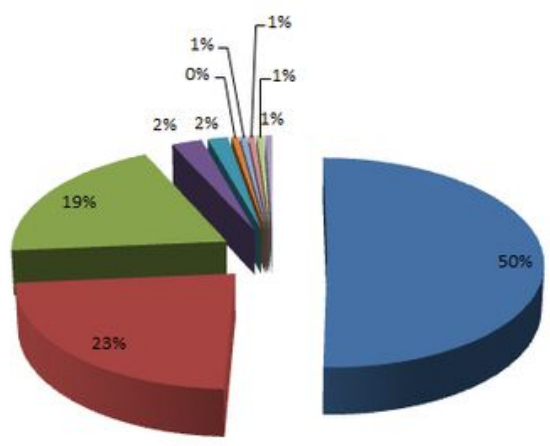

A

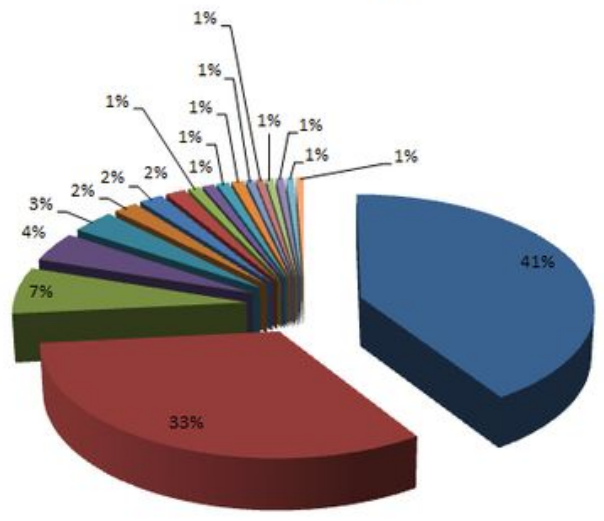

B

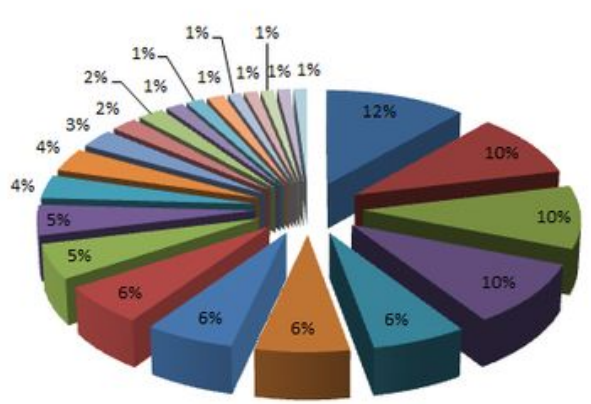

C
" cell

membrane

Extracellular region

m macromolecular complex

m adherens junction

in cell junction

m cell-cell junction

Ex extracellular matrix

II synapse

= integral component of membrane

\section{Winding}

- catalytic activity

m nucleic acid binding

- transporter activity

m structural molecule activity

II ATPase activity, coupled

enzyme inhibitor activity

menzyme activator activity

- immune system process

-1 response to external stimulus

In response to chemical

- response to endoge nous stimulus

macromolecule localization

- cell death

- developmental process

I response to organic substance

- response to metal ion

mbiological adhesion

Figure 10

Gene Ontology (GO) annotations of differentially expressed proteins (DEPs) of Sijunzi decoction intervention spleen deficiency syndrome. (A) CC. (B) MF. (C) BP. 


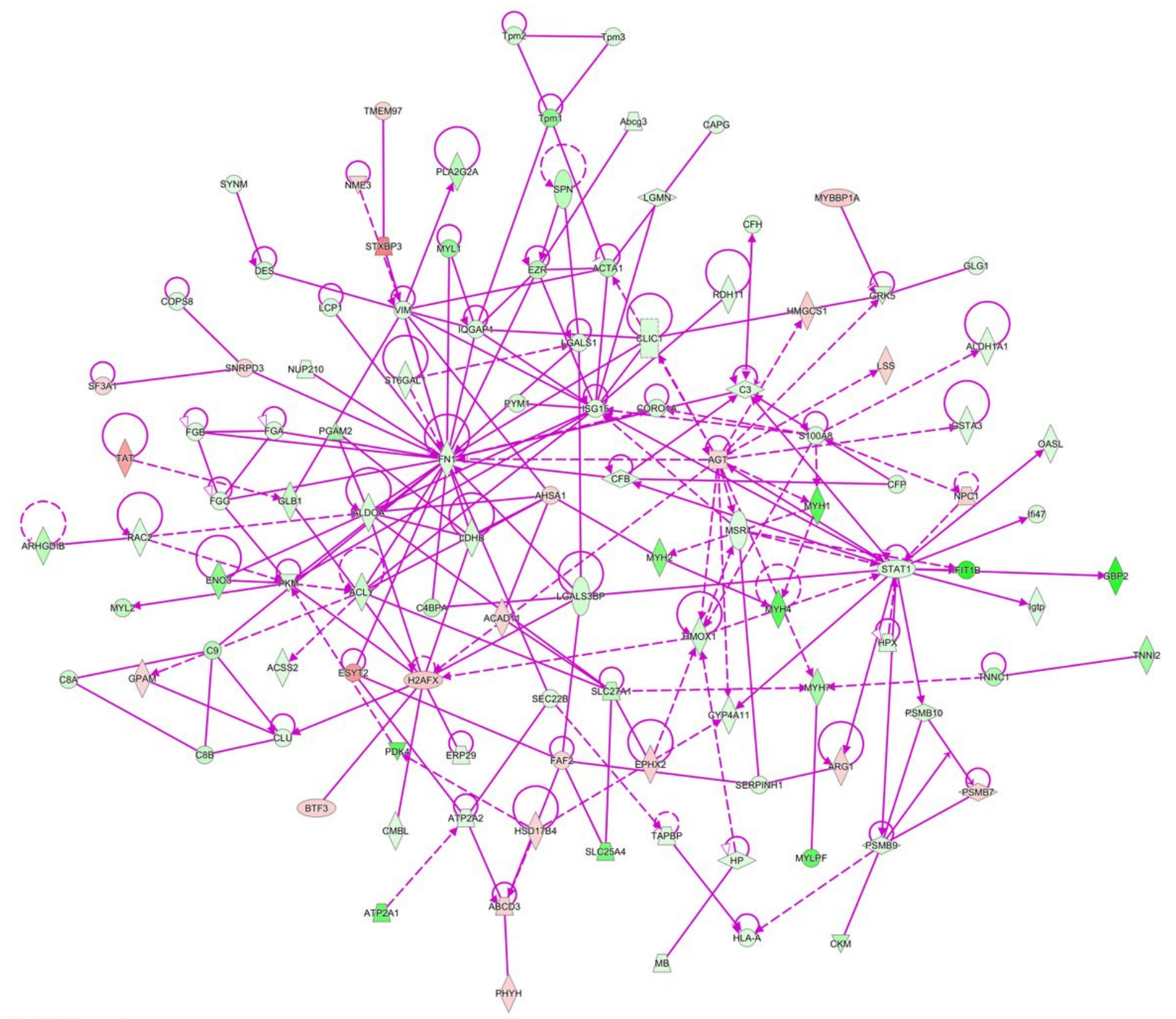

Figure 12

Protein-protein interaction analysis of differentially expressed proteins (DEPs) of Sijunzi decoction intervention spleen deficiency syndrome by ingenuity pathway analysis (IPA). Red represents upregulated proteins, and green represents downreregulated proteins when the model group is compared to the Sijunzi decoction-treated group. 

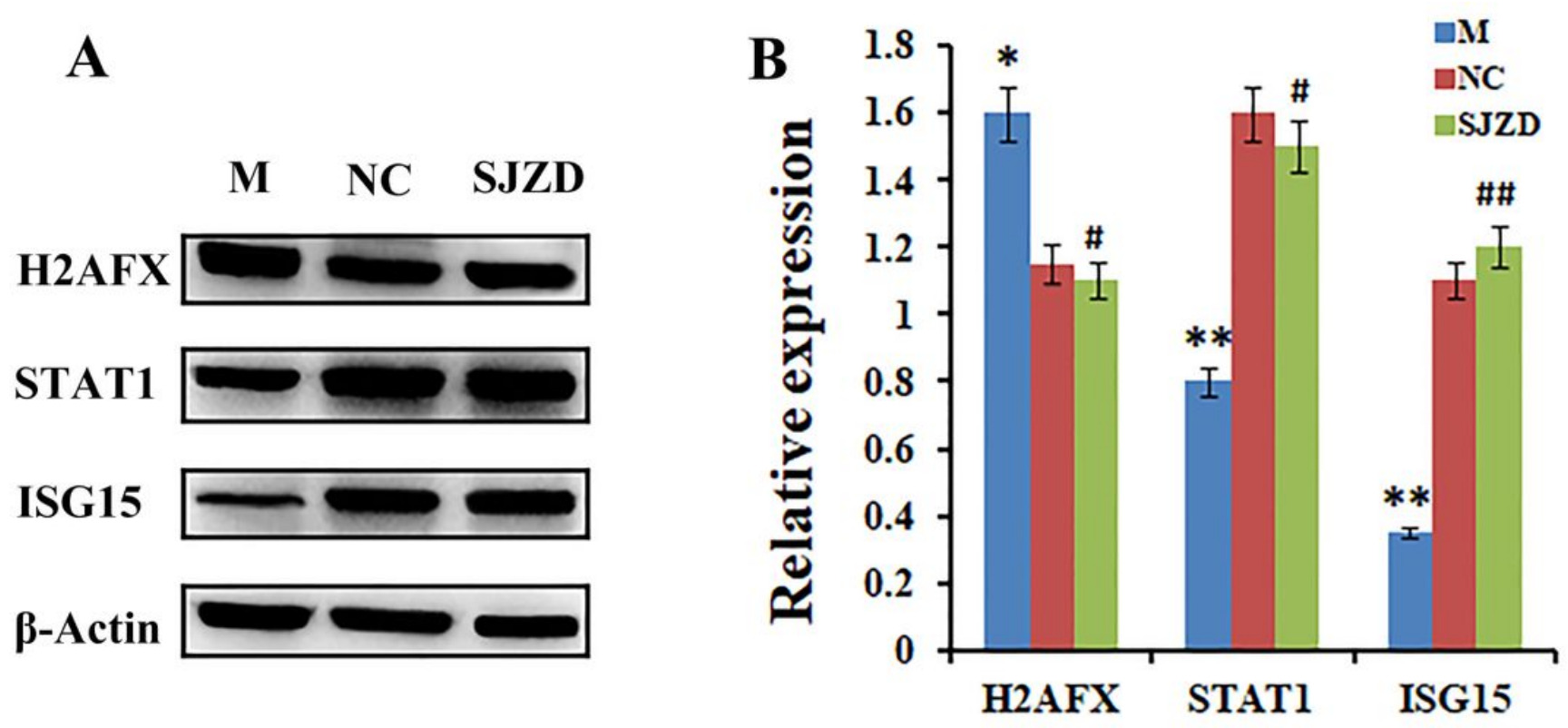

Figure 13

Western blot verification of differentially expressed proteins (DEPs) of H2AFX, STAT1 and ISG15. (A) Western blot results showing expression levels of H2AFX, STAT1 and ISG15 in model, the normal control and Sijunzi decoction-treated groups. (B) Statistical analysis of the gray-level value of the bands after three independent experiments. The protein bands were normalized to $\beta$-actin bands. The data were mean $\pm S D$. * represent significantly different compared to the normal control group ( $p$ value $<0.05$ ); ** represent highly significantly different compared to the normal control group ( $p$ value $<0.01$ ); \# represent significantly different compared to the model group ( $p$ value $<0.05$ ); \#\# represent highly significantly different compared to the model group ( $p$ value $<0.01$ ).

\section{Supplementary Files}

This is a list of supplementary files associated with this preprint. Click to download.

- Additionalfile2.pdf

- Additionalfile1.pdf

- Additionalfile1.pdf

- Additionalfile2.pdf 